\title{
Reply to comment on Bao et al.: Lesser trochanteric osteotomy in total hip arthroplasty for treating CROWE type IV developmental dysplasia of hip
}

\author{
Bao Nirong • Zhao Jianning
}

Received: 15 February 2013 /Accepted: 16 February 2013 /Published online: 7 March 2013

(C) Springer-Verlag Berlin Heidelberg 2013

\section{Dear Editor,}

The comments on our article entitled "Lesser trochanteric osteotomy in total hip arthroplasty for treating CROWE type IV developmental dysplasia of hip" [1] are really appreciated. The replies to the questions are as below.

1. The greater trochanter was carefully protected during the preparation of the femoral canal. The cancellous bone of the greater trochanter is largely removed before the impaction of the rasps into the canal, which minimises the difficulty in insertion into the proximal femur. The lateral wall of the greater trochanter is further protected by one assistant using a hand to slightly push the wall medially. A modular prosthesis was recommended for its better contour design for the proximal femoral canal. Prophylactic cerclage may help sometimes although it is hard to set it in the right position.

2. We agree with the statement that in general the soft tissue tension of the ipsilateral limb is higher after lesser trochanteric osteotomy than that after subtrochanteric osteotomy. Notwithstanding this consideration, the results of follow-up did show the low incidence rate of neurovascular injuries, which is in line with a previous study of the effect of lengthening the limb on the sciatic nerve [2]. In addition, shortening the femur when

B. Nirong $\cdot$ Z. Jianning $(\bowtie)$

Department of Orthopedic Surgery, Nanjing Jinling Hospital, 305

Zhongshan East Road,

Nanjing, China 210002

e-mail: zhaojianning.0207@163.com

Z. Jianning

e-mail: bnrbnr@sina.com performing subtrochanteric osteotomy will cause a leg length discrepancy after surgery, which may be the reason for chronic low back pain [3].

3. Different from subtrochanteric osteotomy, we managed to correct the abnormal anteversion angle of the proximal femur and to insert the stem parallel to the longitudinal axis of the femoral canal cross section. Moreover, the anteversion angle of the acetabular cup needed to be set less than normal. Although no significant correlation was found between post-operative dislocation of the prosthesis and its anteversion angle, we agree with the author that managing combined anteversion angle of the cup and stem into the range from 30 to $45^{\circ}$ would decrease the risk of post-operative dislocation [4]. Overcorrection of anteversion that causes posterior hip dislocation should be avoided especially for those with bilateral hip dysplasia which is usually combined with excessive lumbar lordosis.

\section{References}

1. Bao N, Meng J, Zhou L et al (2013) Lesser trochanteric osteotomy in total hip arthroplasty for treating CROWE type IV developmental dysplasia of hip. Int Orthop. doi:10.1007/s00264-012-1758-4

2. Eggli S, Hankemayer S, Müller ME (1999) Nerve palsy after leg lengthening in total replacement arthroplasty for developmental dysplasia of the hip. J Bone Joint Surg Br 81:843-845

3. Hasegawa Y, Iwase T, Kanoh T et al (2012) Total hip arthroplasty for Crowe type IV developmental dysplasia. J Arthroplasty 27:1629-1635. doi:10.1016/j.arth.2012.02.026

4. Wang L, Trousdale RT, Ai ST et al (2012) Dislocation after total hip arthroplasty among patients with developmental dysplasia of the hip. J Arthroplasty 27:764-769 